\title{
Genomic Organization of the Human Tissue Inhibitor of Metalloproteinases-3 (TIMP3)
}

\author{
Heidi Stöhr, ${ }^{1}$ Kirsten Roomp, ${ }^{2}$ Ute Felbor, ${ }^{1}$ and Bernhard H.F. Weber ${ }^{1,3}$ \\ ${ }^{1}$ Institut für Humangenetik, Universität Würzburg, D-97074 Würzburg, Germany; ${ }^{2}$ Simon Fraser \\ University, Burnaby, British Columbia V5A 156, Canada
}

\begin{abstract}
The tissue inhibitors of metalloproteinases (TIMPs) play a crucial role in the physiological turnover of the extracellular matrix (ECM) by tightly regulating matrix metalloproteinase (MMP) activities. Disturbances in the TIMP/MMP system have been implicated in many disease processes where loss of ECM integrity is a principal feature. More recently, we have shown that mutations in TIMP3 cause the autosomal dominant disorder Sorsby's fundus dystrophy (SFD). This is a macular degeneration disorder with characteristic ECM irregularities in Bruch's membrane. To further facilitate mutational analysis and to provide a basis for functional studies, we now report the genomic organization of the human TIMP3 gene.
\end{abstract}

Cell-cell contacts in multicellular organisms are mediated through the extracellular matrix (ECM), which consists of a highly complex aggregate of secreted proteins and carbohydrates. In response to normal developmental changes and tissue turnover, the components of the ECM are continously subjected to degradation and resynthesis. Disturbances in these dynamic processes may lead to loss of ECM integrity, which is thought to play an important role in many pathologic conditions (Docherty et al. 1992).

The coordinated remodeling of the ECM requires the tightly controlled activity of a number of proteinases, including the family of matrix metalloproteinases (MMPs). These MMPs are able to degrade all components of the ECM and the basement membranes (Matrisian 1990). The regulation of the MMPs occurs at many levels and includes the tissue inhibitors of metalloproteinases (TIMPs). To date, three members of the TIMP gene family have been identified: TIMP1 (Docherty et al. 1985), TIMP2 (Boone et al. 1990), and TIMP3 (Apte et al. 1994a; Silbiger et al. 1994).

The implication of TIMP3 in the pathogenesis of Sorsby's fundus dystrophy (SFD) (Weber et al. 1994) has emphasized further the importance of the inhibitors in ECM homeostasis. SFD is an adult onset macular degeneration characterized by complications arising from ECM disturbances in Bruch's membrane (Capon et al. 1989). How-

\footnotetext{
${ }^{3}$ Corresponding author.

E-MAIL bweb@biozentrum.uni-wuerzburg.de; FAX 49-931. 888-4069.
}

ever, the exact molecular mechanisms by which these mutations cause SFD are still unknown. To facilitate the mutational and functional analysis of TIMP3, we determined the genomic structure, identified the exon/intron sequences of the gene, and analyzed the putative promotor region by an interspecies comparison with the mouse Timp3 promotor.

\section{RESULTS AND DISCUSSION}

\section{Genomic Organization of the Human TIMP3 Gene}

Overlapping retinal TIMP3 CDNA probes were used to screen two flow-sorted chromosome 22specific cosmid libraries. Five independent genomic cosmid clones (I-224, P-118, B11B11, $\mathrm{A} 12 \mathrm{G} 11$, and $\mathrm{D} 8 \mathrm{H} 6$ ) were isolated and assembled into a contig spanning $\sim 100 \mathrm{~kb}$ of genomic DNA (Fig. 1). To identify fragments containing exonic sequences, cDNA probes were hybridized to EcoRI- or Pstl-restricted cosmid DNAs. Three positive EcoRI fragments $(6.5 \mathrm{~kb}$ derived from B11B11; $1.4 \mathrm{~kb}$ and $2.5 \mathrm{~kb}$, both derived from D8H6), as well as a $0.3-$ and a 5.0-kb PstI fragment (both derived from I-224) were subcloned and partially sequenced. Subsequently, alignment of the genomic sequences to the published TIMP3 cDNAs revealed the exon/intron organization of the human TIMP3 gene that is encoded by 5 exons (Fig. 1) with the most likely assignments of donor and acceptor splice junctions following the 5'-GT-AG-3' rule (Breathnach et al. 1978).

Exon 1 contains the translation initiation start codon ATG as well as 280 bp of upstream 
STÖHR ET AL.
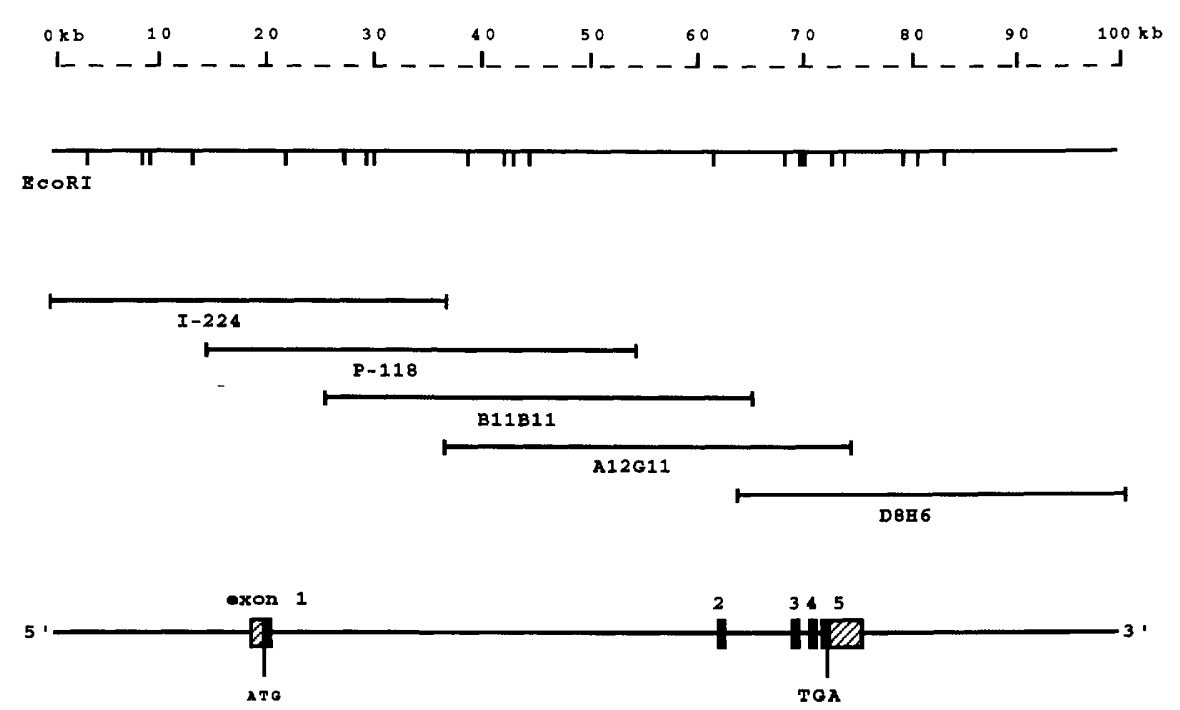

Figure 1 Structure of the human TIMP-3 gene. The genomic organization was deduced from five overlapping genomic clones (1-224, P-118, B11B11, $\mathrm{A} 12 \mathrm{G} 11$, and D8H6) indicated by solid lines. EcoRI restriction sites within the genomic clones are shown as solid bars and the size of the contig is represented as a broken line above the restriction map. The relative positions of the 5 exons are based on their location in the respective EcoRl fragment and are shown below the cosmid contig. Coding exons are indicated by solid boxes, $5^{\prime}$ - and 3 '-flanking regions by hatched boxes, and introns by solid lines.

sequence corresponding to the most $5^{\prime}$-extending cDNA isolated (Silbiger et al. 1994). This suggests that the 5'-flanking region of the TIMP3 gene is not interrupted further by intervening sequences. Exons 2-4 are 84, 112, and 122 bp, respectively. The $2.5-\mathrm{kb} E c o$ RI fragment from cosmid clone D8H6 contains exon 5 with the translation stop codon TGA and an additional $1057 \mathrm{bp}$ of 3'-untranslated region. An EcoRI restriction site $1057 \mathrm{bp}$ downstream of the stop codon is also present in published TIMP3 cDNAs (Byrne et al. 1995).
The overall organization of the human TIMP3 gene seems very similar to that of the recently reported murine homolog (Apte et al. 1995). A comparison between human and murine exon/intron splice junctions reveals that the coding regions in both species are interrupted by intervening sequences at identical positions within the respective triplet codons. In addition, a similar conservation of the relative locations of the exon/intron boundaries has also been reported for the murine Timp3 and Timp1 genes (Apte et al. 1995). This suggests that the TIMP gene family might have evolved from a common ancestor and might have arisen by duplication of an ancient precursor TIMP locus.

\section{Comparison of the 5'-flanking Regions of the Human and Mouse TIMP3 Genes}

Assuming very similar functions of the highly homologous TIMP3 genes in human and mouse (Silbiger et al. 1994; Leco et al. 1994), the regulation of TIMP3 expression should be subjected to strong evolutionary constraints for preservation of putative regulatory elements. Therefore, we compared $\sim 1200$ bp of the $5^{\prime}$-flanking region of

Figure 2 Comparison between the $5^{\prime}$ flanking regions of human and murine TIMP3 gene. (A) Schematic representation of the $G+C$ content and frequency of $C p G$ dinucleotides across the coding region of exon 1 and $1184 \mathrm{bp}$ of the $5^{\prime}$-flanking region of the human and murine TIMP3 genes. The $\mathrm{G}+\mathrm{C}$ composition is indicated by a broken line; the observed versus expected frequency of $\mathrm{CpC}$ (obs/exp CpG) dinucleotides by a solid line along the sequence. Human and murine $\mathrm{CpG}$ islands that fulfill the criteria of moving averages of $>50 \% \mathrm{G}+\mathrm{C}$ and a value of $>0.6$ for observed versus expected frequency of $\mathrm{CpG}$ dinucleotides (Gardiner-Garden and Frommer 1987) are shown by thick bars above the graphs. Below the plot, the relative position of the coding region of exon 1 is indicated as a solid box, indicating the translation start codon. The solid line represents $5^{\prime}$-flanking sequence, and the numbers below correspond to base pairs relative to the start codon ATG, with A being +1 . $(B)$ BESTFIT alignment of the nucleotide sequences of the putative promotor regions of human and murine TIMP3. Sequence data for the mouse gene are taken from Sun et al. (1995). The translation codon is marked by a solid box. The asterisks above the human and below the murine sequence denote the beginning of the longest isolated CDNA clones in the corresponding species (Leco et al. 1994; Byrne et al. 1995); the solid circle below the nucleotide adenine at position -316 indicates the major transcription start site of the mouse Timp3 gene (Sun et al. 1995). The $G+C$-rich cluster with significant sequence similarity between human and mouse as well as the conserved putative binding sites for SP1 and a possible TATA box are boxed. 


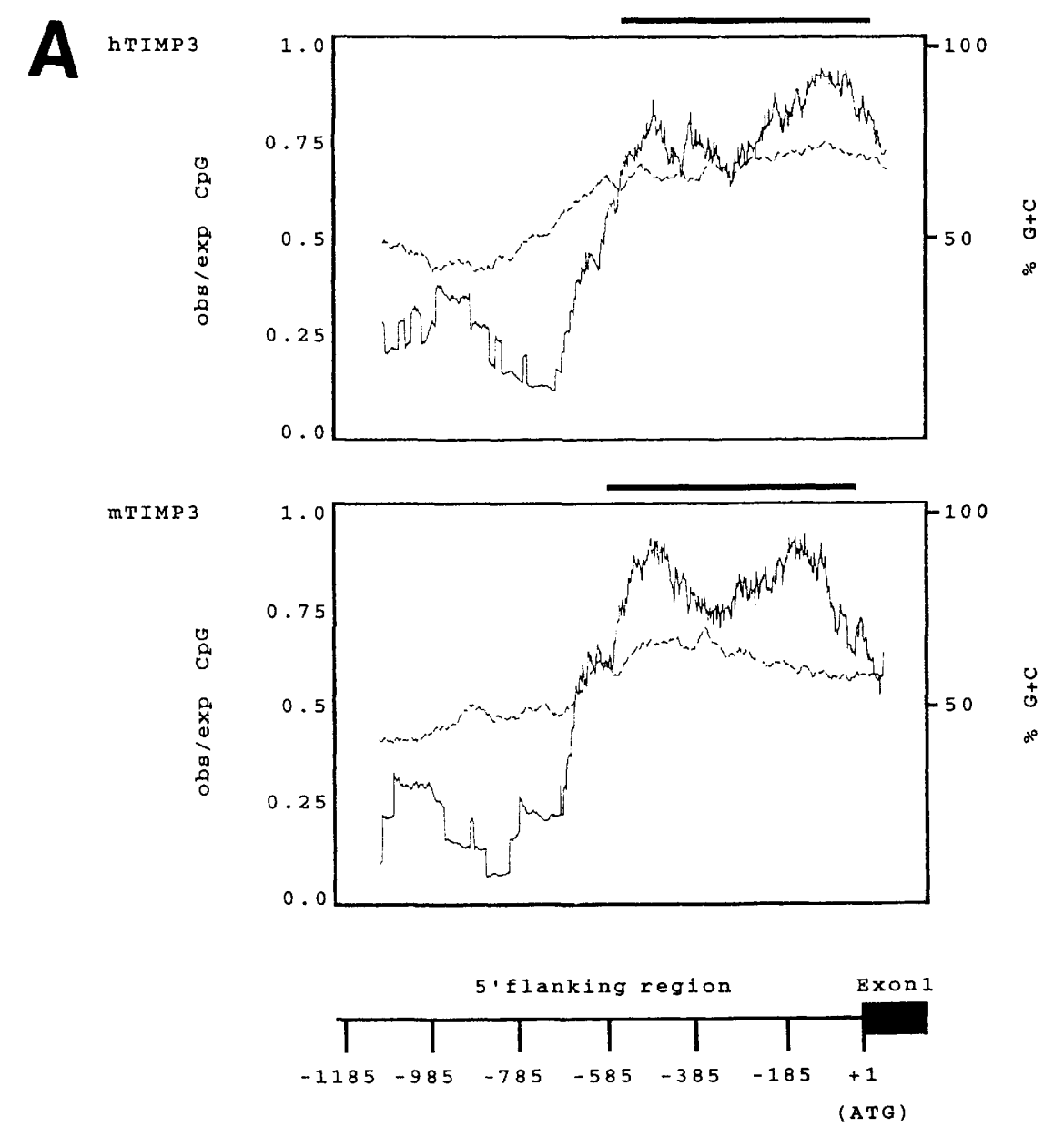

B

TTTCTCCTCTNTNCCTGCTCTCTCCAGAGAAACTGGAGGGGTAGCAGTT. .

. AGCATTCCCCC... GCTGGTTCCACCAAGCACAGTCAAGGTC.TC -706

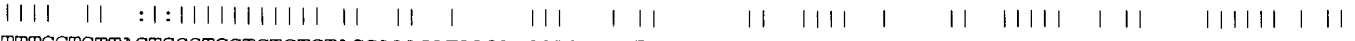
TTTCCTCTTACTCCCTGCTCTCTCTAGGAAAGATAAGAAGGTGAAATTTCTTTGAGGCTTCCTTCTTGACTTTTTCCAGGAGGCGTGATCAAGGACTTC -685

TAGGACATGGCCACCCCTCACCTGTGGAAGCGGTCCTGCTG. . GGGTGGGTGGGTGTTAGTTGGTTCTGGTTTGGGTCAGAGACACCCAGTGGCCCAGG -609

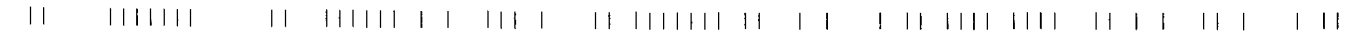
AGGGTGCGGGCCACCTGGGGCCCTTGGAAGAGATTATGCAGGAAGCCTGGGTGGCTGGGACTGAATCCTTGTTTTAGGTCCAAGGCTCATAGAGAGGC.GG -586

TGGGCGTGGGGCCAGGGCGCAGACGAGAAGGGGCACGAGGGCTCCGCTCCGAGGACCCAGCGGCAAGCACCGGTCCCGGGNGGG CCCAGCCCACCCACT-509 $11111111111 \quad 11111111 \quad 11111111 \quad 111 \quad 11111111111: 1|| 11111111111 \mid$ TGGGCGTAGGGC ..............GAAGGGGCCGTGGGACGCCGCT ........... GTAGCGCACACCGG. CCCAGGACGCdCCCAGCCCACACCCT -512

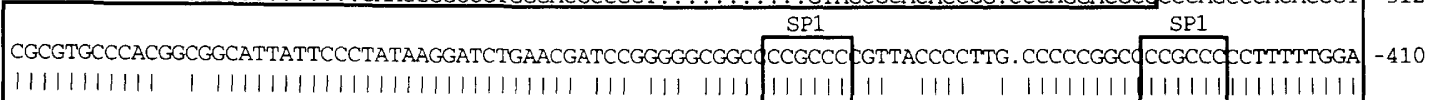
CGCGTGCCCACAACTGCATTATTCCCTATAAGGATCTGAACGGTCC. GGGCCGGC LCCGCCCFGTCGCCCCGCGCCCCCCGGCQCCGCCOCCTTTTTGGA - 413 GGGCCGATGAGGTAATGCGGCTCTGCCATTGGTCTGAG SPGGCE 1111111111111111111111111111111111111111111011111 1 1111 111 11111111 | 1111 1 $11111|11111|$ GGGCCGATGAGGTAATGCGGCGCTGCCATTGGTCGGAGdGGGCGA ACCCCGAGCTCCCGAGGGGCTGGGTCCCCTCGCGCCC . . . CATATHACTCTGC - 317 CGCC aGGCAGACGGCGCAGAGCGGGCAGCAGGCAGGCGGCGGGCGCTCAGACGGCTTCTCCTCCTCCTCTTGCTCCTCCAGCTCCTGCTCCTTCGCCGG -212

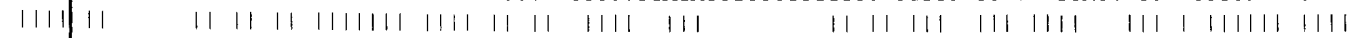
$\underset{\star}{A . G C C O T G G A C T C T C G C T C A C A G T G G G C A G C G G G C A . ~ G C C G C C A G C G C C A A G A . . . . . . . ~ A T C T T C T T C T C C C G C T T C T C C G C T T C C C G A T C C T T C T C C G G ~-225 ~}$

GAGGCCGCCCGCCGAGTCCTGGCCAGCGCCGAGGCAGCCTGCCTGCGCCCC. . A.TCCCGTCCCGCCGGGC. ACTCGGAGGGCAGCGCGCCGGAGGCCAA -116

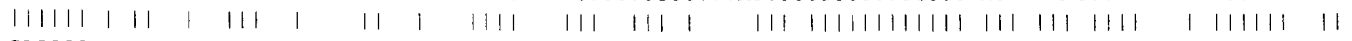
GAGGCCACTCGTTGGCTCCGCGGACTCG. TGTTCCAGCGACCCTTGGCCACTTAGTCCTGTCCCGCCGGGCTACTTGGAAGGCACTTCCCCGGAGCTCAT -126 GGTTGCCC ..... CGCACGGCCCGG ..... CGGGCGAGCGAGCTCGGGCTGCAGCAGCCCCGCCGGCGCGCACGGCAACTTTGGAGAGGCGAGCAGCA -28

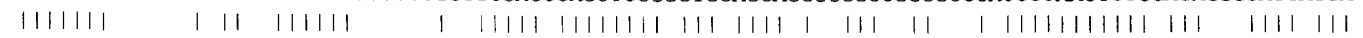
CGTTGCCCACCGTGCACAGTGCCCGGTTAAACCCAGCGAGTGAGCTCGGACTGTAGCATCAGCGCTAGC . . CTCGGCAACTTTGAAGAAAAGAGCGGCA -29 $+1$

GCCCCGGCAGCGG. CGGCAGCAGCGGCAATGACCCCTTGGCTCGGGCTCATCGTGCTCCTGGGCAGCTGGAGCCTGGGGGACTGGGGCGCCGAGGCGTGC +72

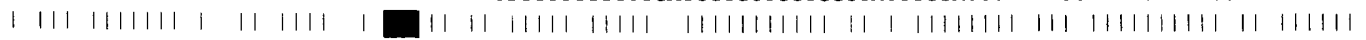
GTCCCCGCAGCGGACCACAACAGCTACCATGACTCCCTGGCTTGGGCTTGTCGTGCTCCTGAGCTGTTGGAGCCTTGGGCACTGGGGCGCGGAAGCGTGC +72

Figure 2 (See facing page for legend.) 


\section{STÖHR EI AL.}

human TIMP3 with published mouse Timp3 sequences (Sun et al. 1995).

$\mathrm{G}+\mathrm{C}$ content and observed versus expected frequency of $\mathrm{CpG}$ dinucleotides were determined along the entire $1184 \mathrm{bp}$ of the 5 '-flanking region of mouse and human TIMP3 (Fig. 2A). The segments around nucleotides +1 to -580 , especially, show high averages of $>50 \% \mathrm{G}+\mathrm{C}$ and values of $>0.6$ for observed versus expected frequency of $\mathrm{CpG}$ dinucleotides, thus, fulfilling the criteria for the presence of true CPG islands in both species (Larsen et al. 1992) (Fig. 2A). Outside of this region, around nucleotides -1184 to -580 , is a sharp decline in both the $G+C$ content as well as in the observed versus expected $\mathrm{CpG}$ frequency similar to the observed suppression of CpGs in bulk genomic DNA (Fig. 2A).

Alignment of the putative human and mouse TIMP3 promotor regions revealed an overall sequence similarity of 75\% (Fig. 2B). Significant sequence similarities were found in the area around nucleotides -522 to -307 . In this stretch of DNA, we identified also three conserved putative Sp1 consensus binding sites at positions -452 to $-447,-425$ to -420 , and -370 to -365 and a conserved TATA box at position -324 to -320 (numbers are relative to the human TIMP3 start codon). The longest human TIMP3 cDNA clone isolated starts at nucleotide -283 (Byrne et al. 1995), whereas the longest mouse cDNA begins at nucleotide -309 (Leco et al. 1994). A major transcription initiation site at position -316 has been defined for the mouse Timp3 gene (Sun et al. 1995) (Fig. 2B). Other putative transcription factor binding sites (additional SP1 and AP1, PEA3, CTF/NF-1) were identified but did not appear to be highly conserved between human and mouse populations.

The presence of a potential TATA box in TIMP3 is interesting in light of the fact that in the other two family members-murine Timp1 (Edwards et al. 1992) and human TIMP2 (DeClerck et al. 1994)-no such canonical TATA box has been identified. It has been suggested that TIMP1 and TIMP2 belong to the TATA box-deficient, $\mathrm{G}+\mathrm{C}$-rich promotor group (Edwards et al. 1992; DeClerck et al. 1994), which previously has been found to direct transcription of housekeeping genes (Dynan 1986), although in some cases tissue-specific transcription may be possible also (Oliva et al. 1991). TIMP3 expression appears to be different from TIMP1 and TIMP2 in that it has been detected in many organs but seems to be restricted to certain tissue components, for exam- ple, the epithelial cells (Apte et al. 1994b). Thus, TIMP3 may be considered an intermediate between constitutively expressed genes and tissueor cell-specific expressed genes.

Taken together, we have determined the gene structure of human TIMP3 and have shown that the 5 exons of this gene span $\sim 55 \mathrm{~kb}$ of genomic DNA. We have provided sequence information on all exon/intron boundaries that will greatly facilitate the mutational analysis of TIMP3 in human genetic diseases. Besides its involvement in SFD, TIMP3 might also play a critical role in other human retinopathies (Jones et al. 1994), as well as in some debilitating disorders where loss of ECM integrity is a principal feature (Docherty et al. 1992). In addition, we have sequenced $1200 \mathrm{bp}$ of the $5^{\prime}$-flanking region of the human TIMP3 gene and, by sequence alignment to the homologous region in mouse (Sun et al. 1995), have identified several highly conserved putative regulatory elements. Although these sites will have to be confirmed by binding assays and functional studies, those predictions may greatly facilitate the further characterization of the TIMP3 promotor region.

\section{METHODS}

For library screening TIMP3 CDNA probes were obtained by RT-PCR amplification of total human retinal RNA with three primer pairs designed according to published TIMP3 CDNA sequences (Silbiger et al. 1994). Primers used were 5'TIMP (5'-TTCTCCTCCTCCTCTTGC-3')/1FF (5'ACGATGTCGGAGTTGCA-3'), 1F (5'-TGGAGCCTGGGGGACTGG-3')/1R (5'- CTGGGAGAGGGTGAGCTGG-3'), and $2 \mathrm{~F}\left(5^{\prime}\right.$ - CAACTTCGTGGAGAGGTGGG-3')/2R (5'AGGGTCTGGCGCTCAGGG-3'). High-density gridded filters of chromosome 22-specific cosmid libraries were kindly provided by the Reference Library Data Base of the Imperial Cancer Research Fund, London, UK (constructed in Lawrist 4) and by Peter Scambler and Sara C.M. Daw, Institute of Child Health, London, UK (constructed in Lawrist 16 by the Lawrence Livermore National Laboratories, Livermore, CA).

Overlaps between positive clones were detected by Southern blot hybridizations of T3-, SP6-, and T7-endclone fragments to EcoRI-restricted digests of the cosmid DNAs. Exon-containing cosmid fragments were subcloned into the plasmid vector pBluescript SK(-) (Stratagene). Doublestranded sequencing was carried out using the dideoxy chain termination method with vector-specific T3 and T7 primers as well as internal exon-specific primers 5'TIMP, $1 F F, 1 F, 1 R, 2 F$, and $2 R$. Exon/intron boundaries were identified by alignment of the genomic sequence with the published TIMP3 cDNA (Silbiger et al. 1994) using MacVector sequence analysis software (release 4.0 ). The identified splice sequences were in excellent agreement with published consensus sequences. The distribution of $\mathrm{G} / \mathrm{C}$ content and observed versus expected frequency of $\mathrm{CpG}$ dinucleotides along the human and mouse $5^{\prime}$ flanking se- 


\section{GENE STRUCIURE OF TIMP3}

quence was plotted using the Wisconsin GCG package (version 8,1994 ). For a window size of 200 bp the criteria for a $C p G$ island are $C+G>50 \%$ and observed versus expected $\mathrm{CpG}>0.6$. Optimal alignment of the putative human and mouse promotor regions were achieved by using the GCG program BESTFIT.

\section{ACKOWLEDGMENTS}

We thank Manfred Gessler for his critical suggestions on the manuscript.

The publication costs of this article were defrayed in part by payment of page charges. This article must therefore be hereby marked "advertisement" in accordance with 18 USC section 1734 solely to indicate this fact.

\section{Note}

The nucleotide sequences reported in this paper have been submitted to the GenBank/EMBL data bank under accession numbers U33110, U33111, U33112, U33113, and U33114.

\section{REFERENCES}

Apte, S.S., M.-G. Mattei, and B.R. Olsen. 1994a. Cloning of the cDNA encoding human tissue inhibitor of metalloproteinases-3 (TIMP-3) and mapping of the TIMP3 gene to chromosome 22. Genomics 19: 86-90.

Apte, S.S., B.R. Olsen, and G. Murphy. 1995. The gene structure of tissue inhibitor of metalloproteinases (TIMP-3) and its inhibitory activities define the distinct TIMP gene family. J. Biol. Chem. 270: 14313-14318.

Apte, S.S., K. Hayashi, M.F. Seldin, M.-G. Mattei, M. Hayashi, and B.R. Olsen. 1994b. Gene encoding a novel murine tissue inhibitor of metalloproteinases (TIMP), TIMP-3, is expressed in developing mouse epithelia, cartilage, and muscle, and is located on mouse chromosome 10. Dev. Dynam. 200: 177-197.

Boone, T., M.J. Johnson, Y.A. DeClerck, and K.E. Langley. 1990. cDNA cloning and expression of a metalloproteinase inhibitor related to tissue inhibitor of metalloproteinases. Proc. Natl. Acad. Sci. 87: 2800-2804.

Breathnach, R., C. Benoist, K. O'Hare, F. Gannon, and P. Chambon. 1978. Ovalbumin gene: Evidence for a leader sequence in mRNA and DNA sequences at the exon-intron boundaries. Proc. Natl. Acad. Sci. 75: 4853-4857.

Byrne, J.A., C. Tomasetto, N. Rouyer, J.-P. Bellocq, M.-C. Rio, and P. Basset. 1995. The tissue inhibitor of metalloproteinases- 3 gene in breast carcinoma: Identification of multiple polyadenylation sites and a stromal pattern of expression. Mol. Med. 1: 418-427.

Capon, M.R.C., J. Marshall, J.I. Krafft, R.A. Alexander, P.S. Hiscott, and A.C. Bird. 1989. Sorsby's fundus dystrophy: A light and electron microscopic study. Ophthalmology 96: 1769-1777.
DeClerck, Y.A., M.I. Darville, Y. Eeckhout, and G.G. Rousseau. 1994. Characterization of the promoter of the gene encoding human tissue inhibitor of metalloproteinases-2 (TIMP-2). Gene 139: 185-191.

Docherty, A.J.P., A. Lyons, B.J. Smith, E.M. Wright, P.W. Stephens, T.J. Harris, G. Murphy, and J.J. Reynolds. 1985. Sequence of human tissue inhibitor of metalloproteinases and its identity to erythroid-potentiating activity. Nature 318: 66-69.

Docherty, A.J.P., J. O'Connell, T. Crabbe, S. Angal, and G. Murphy. 1992. The matrix metalloproteinases and their natural inhibitors: Prospects for treating degenerative tissue diseases. Trends Biotechnol. 10: $200-207$.

Dynan, W.S. 1986. Promotors for housekeeping genes. Trends Genet 2: 196-197.

Edwards, D.R., H. Rocheleau, R.R. Sharma, A.J. Wills, A. Cowie, J.A. Hassell, and J.K. Heath. 1992. Involvement of AP1 and PEA3 binding sites in the regulation of murine tissue inhibitor of metalloproteinases-1 (TIMP-1) transcription. Biochim. Biophys. Acta 1171: 41-55.

Gardiner-Garden, M. and M. Frommer. 1987. CpG islands in vertebrate genomes. J. Biol. Chem. 196: $261-282$.

Jones, S.E., C. Jomary, and M.J. Neal. 1994. Expression of TIMP3 mRNA is elevated in retinas affected by simplex retinitis pigmentosa. FEBS Lett. 352: 171-174.

Larsen, F., G. Gundersen, R. Lopez, and H. Prydz. 1992. $\mathrm{CpG}$ islands as gene markers in the human genome. Genomics 13: 1095-1107.

Leco, K.J., R. Khokha, N. Pavloff, S.P. Hawkes, and D.R. Edwards. 1994. Tissue inhibitor of metalloproteinases-3 (TIMP-3) is an extracellular matrix-associated protein with a distinctive pattern of expression in mouse cells and tissues. J. Biol. Chem. 269: 9352-9360.

Matrisian, L.M. 1990. Metalloproteinases and their inhibitors in matrix remodeling. Trends Genet. 6: $121-125$.

Oliva, D., L. Cali, S. Feo, and A. Giallongo. 1991. Complete structure of the human gene encoding neuron-specific enolase. Genomics 10: 157-165.

Silbiger, S.M., V.L. Jacobson, R.L. Cupples, and R.A. Koski. 1994. Cloning of cDNAs encoding human TIMP-3, a novel member of the tissue inhibitor of metalloproteinase family. Gene 141: 293-297.

Sun, Y., G. Hegamyer, H. Kim, K. Sithanandam, H. Li, R. Watts, and N.H. Colburn. 1995. Molecular cloning of mouse tissue inhibitor of metalloproteinases- 3 and its promotor. J. Biol. Chem. 270: 19312-19319.

Weber, B.H.F., G. Vogt, R.C. Pruett, H. Stöhr, and U. Felbor. 1994. Mutations in the tissue inhibitor of metalloproteinases-3 (TIMP3) in patients with Sorsby's fundus dystrophy. Nature Genet. 8: 352-356.

Received August 29, 1995; accepted in revised form November 21, 1995. 


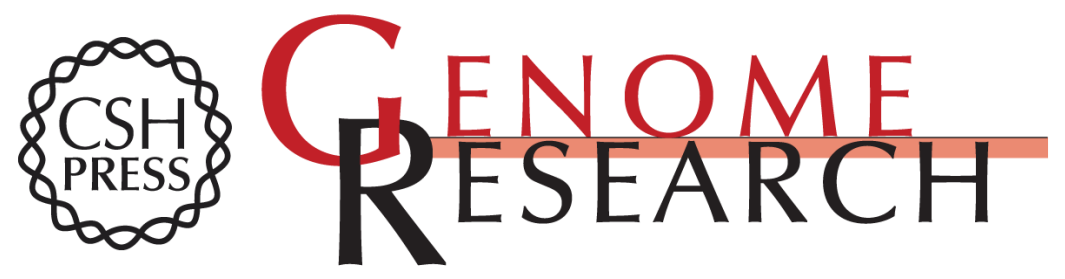

\section{Genomic organization of the human tissue inhibitor of metalloproteinases-3 (TIMP3)}

H Stöhr, K Roomp, U Felbor, et al.

Genome Res. 1995 5: 483-487

Access the most recent version at doi:10.1101/gr.5.5.483

References This article cites 21 articles, 5 of which can be accessed free at:

http://genome.cshlp.org/content/5/5/483.full.html\#ref-list-1

\section{License}

Email Alerting Receive free email alerts when new articles cite this article - sign up in the box at the Service top right corner of the article or click here.

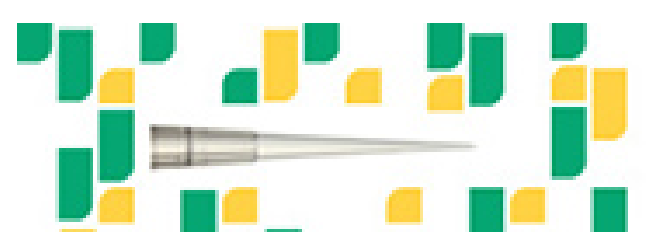

To subscribe to Genome Research go to: https://genome.cshlp.org/subscriptions 